Check for updates

Cite this: RSC Adv., 2019, 9, 17612

Received 11th April 2019 Accepted 29th May 2019

DOI: $10.1039 / c 9 r a 02729 g$

rsc.li/rsc-advances

\section{The influence of three acid modifications on the physicochemical characteristics of tea-waste biochar pyrolyzed at different temperatures: a comparative study $\dagger$}

\begin{abstract}
Chathuri Peiris, (D) ab Oshani Nayanathara, ${ }^{a}$ Chanaka M. Navarathna, (D) Yohan Jayawardhana, ${ }^{d}$ Samadhi Nawalage, (D) a Griffin Burk, ${ }^{c}$ Akila G. Karunanayake, Sunith B. Madduri, ${ }^{f}$ Meththika Vithanage, ${ }^{9}$ M. N. Kaumal, ${ }^{b}$ Todd E. Mlsna, ${ }^{c}$

El Barbary Hassan, ${ }^{f}$ Sachith Abeysundara, ${ }^{\text {h }}$ Felio Ferez and Sameera R. Gunatilake (DD *a

Tea-waste is an abundant feedstock for producing biochar (BC) which is considered to be a cost effective carbonaceous adsorbent useful for water remediation and soil amendment purposes. In the present study, tea-waste BC (TWBC) produced at three different temperatures were subjected to nitric, sulfuric and hydrochloric acid modifications (abbreviated as NM, SM and HM respectively). Characteristics of the raw and modified BC such as ultimate and proximate analyses, surface morphology, surface acidity and functionality, point of zero charge, cation exchange capacity (CEC) and thermal stability were compared to evaluate the influence of pyrolysis temperature and of modifications incorporated. The amount of carboxylic and phenolic surface functionalities on TWBC was seen to decrease by $93.44 \%$ and $81.06 \%$ respectively when the pyrolysis temperature was increased from 300 to $700{ }^{\circ} \mathrm{C}$. Additionally, the yield of BC was seen to decrease by $46 \%$ upon the latter temperature increment. The elemental analysis results provided justification for hightemperature BC being more hydrophobic as was observed by the $61 \%$ increase in $\mathrm{H} / \mathrm{C}$ ratio which is an indication of augmented aromatization. The CEC was the highest for the low-temperature BC and was seen to further increase by NM which is attributed to the $81.89 \%$ increase in carboxylic functionalities. The surface area was seen to significantly increase for BC700 upon NM ( 27 times). The SM led to pore wall destruction which was observed in scanning electron microscopy images. Findings would enable the rational use of these particular modifications in relevant remediation and soil amendment applications.
\end{abstract}

\section{Introduction}

Biochar (BC) is a ubiquitous carbonaceous adsorbent that is gaining colossal research interest worldwide. ${ }^{1}$ Two major

${ }^{a}$ College of Chemical Sciences, Institute of Chemistry Ceylon, Rajagiriya, CO 10107, Sri Lanka.E-mail: ranmal@ichemc.edu.lk

${ }^{b}$ Department of Chemistry, University of Colombo, CO 00300, Sri Lanka

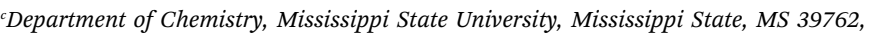
USA

${ }^{d}$ National Institute of Fundamental Studies, Hantana, KY 20022, Sri Lanka

${ }^{\circ}$ Biochar Supreme LLC, Everson, WA 98247, USA

${ }^{f}$ Department of Sustainable Bioproducts, Mississippi State University, Mississippi State, MS 39762, USA

${ }^{g}$ Ecosphere Resilience Research Center, Faculty of Applied Sciences, University of Sri Jayewardenepura, Nugegoda, CO 10250, Sri Lanka

${ }^{h}$ Department of Statistics and Computer Science, University of Peradeniya, Peradeniya, KY 20400, Sri Lanka

${ }^{i}$ Material Science Lab, Integrated Microscopy Center, University of Memphis, Memphis, TN 38152, USA

$\dagger$ Electronic supplementary information (ESI) available. See DOI: $10.1039 / \mathrm{c} 9 \mathrm{ra} 02729 \mathrm{~g}$ commercial applications of $\mathrm{BC}$ can be considered as water remediation and agricultural soil amendment. ${ }^{2-6}$ Production of $\mathrm{BC}$ helps to control environmental pollution by facilitating solid waste management of biomass, while its carbon negative process has been suggested as a means to combat climate change. ${ }^{7}$ It has rapidly gained attention in recent years due to its cost effectiveness and excellent adsorption properties. ${ }^{\mathbf{8}, 9}$ Selection of the feedstock is a critical factor in BC production as the feedstock should be inexpensive and readily available for large scale industrial applications., ${ }^{\mathbf{1 , 8}}$

Numerous physical and chemical properties govern the sorption efficiency of a BC. Physical characterizations include surface morphology, surface area, pore size, ash and moisture content whereas factors such as surface functionality, surface acidity, point of zero charge (PZC) and cation exchange capacity (CEC) etc. can be determined chemically. ${ }^{\mathbf{4 , 1 0}}$ These characteristics mainly depend on the feedstock type and pyrolysis conditions. ${ }^{11}$ Higher efficiencies can be obtained by selecting the correct pyrolysis temperature as well as by providing the optimum sorption conditions. Thus, a proper understanding of 
the $\mathrm{BC}$ of interest becomes essential for it to be used in relevant applications. $^{8}$

The CEC of BC is a vital factor that governs the ability of an adsorbent to retain positively charged ions on its surface. ${ }^{\mathbf{1 2}}$ Properties such as specific surface area, elemental composition and the working $\mathrm{pH}$ of the medium can be considered as aspects that influence the CEC. ${ }^{\mathbf{1 3}, 14}$ The presence of oxygenated surface functional groups (O-SFGs) affect retention capacity significantly as well whilst contributing to the sorption mechanism. These acidic O-SFGs on the BC surface are of three types based on acid dissociation constants; stronger acids (carboxylics; $\mathrm{p} K_{\mathrm{a}} \sim 5-6.4$ ), moderate acids (low $\mathrm{p} K_{\mathrm{a}}$ phenols and the hydrolysis products of lactones; $\mathrm{p} K_{\mathrm{a}} \sim 6$ 6.4-10.3) and weaker acids (high $\mathrm{p} K_{\mathrm{a}}$ phenols; $\left.\mathrm{p} K_{\mathrm{a}} \sim 10.3-13\right) .{ }^{13,15}$ Yet another feature that defines BC functioning is PZC, a property that describes the $\mathrm{pH}$ at which the $\mathrm{BC}$ surface holds a zero net electric charge. ${ }^{\mathbf{1 6}}$ When in a medium that has a $\mathrm{pH}$ higher than the PZC $\left(\mathrm{pH}_{\text {medium }}>\mathrm{pH}_{\mathrm{PZC}}\right)$, the $\mathrm{BC}$ surface is said to possess a predominance of negative charge and vice versa. ${ }^{17}$ The PZC gives an idea as to whether the surface functional groups (SFGs) are in their protonated or deprotonated form.

Far from the latter, for adsorption via pore filling, it is necessary that the pore size of the $\mathrm{BC}$ is compatible with the size of the adsorbate. It has been reported that the sorption within pores occur only when the pore size is at least 1.7 times larger than the second widest dimension of the adsorbate of interest. ${ }^{8}$

Conducting an acid treatment influences the physicochemical properties of BC significantly as it can affect both surface functionality and the porous structure of the $\mathrm{BC} .{ }^{\mathbf{1 8 , 1 9}}$ The pore wall destruction that results as a consequence of acid modification facilitates the pore filling mechanism for different adsorbates..$^{\mathbf{2 0} 21}$ There is also an increase in specific surface area and total pore volume due to demineralization in addition to the increase in surface heterogeneity. ${ }^{22}$ Furthermore, the surface oxidation of the carbonaceous surface introduces new O-SFGs whilst oxidizing existing functional groups. ${ }^{23}$

Nitric acid modification (NM) causes functional group fixation via nitration and oxidation, which introduces nitrogen moieties on BC surfaces via electrophilic substitution..$^{22,24,25}$ Sulfuric acid modification (SM) converts micropores to mesopores and macropores through pore wall destruction, while also acting as a strong oxidizing agent. ${ }^{20}$ Hydrochloric acid modification (HM) on the other hand causes enhancement of O-SFGs via the reduction of carbonyl groups such as lactones, phenols and ethers. ${ }^{24}$

Tea is one of the largest agricultural exports in many tropical and sub-tropical countries including China, Sri Lanka, Kenya, India, Indonesia, Vietnam etc. ${ }^{\mathbf{2 6 , 2 7}}$ The world annual tea production was as high as 5.73 million tons in $2016^{28}$ and it reportedly lead to the accumulation of large amounts of waste. ${ }^{29}$ For example, in Turkey, the rejected tea amounted to about $18 \%$ of total production. ${ }^{30}$ The effective management of this solid waste becomes important. Therefore, tea-waste is an abundant and low-cost feedstock for BC production in these countries. ${ }^{30,31}$ Tea leaves are porous material having a network like structure. ${ }^{31}$ Biochar produced from tea-waste contains $\sim 30 \%$ holocellulose, $\sim 25 \%$ lignin, and $\sim 15 \%$ extractives. ${ }^{32}$ Tea-waste biochar (TWBC) has become a popular sorbent during the past decade due to the presence of these constituents that add functionalities which enhances the sorptive properties of the BC..$^{30-32}$

To the extent of our knowledge, there has been no extensive study carried out to establish a comparison for the influence in physicochemical properties that take place upon the incorporation of common acid modifications. The presented study is an attempt to fill this research gap by conducting a thorough analysis of characteristics related to both the raw and modified $\mathrm{BC}$ produced under three pyrolysis temperatures.

The precursor $\mathrm{BC}$ types produced at 300,500 , and $700{ }^{\circ} \mathrm{C}$ were subjected to NM, SM and HM and the variations in the surface morphology, acidity and functionality, proximate and ultimate analyses parameters, PZC, CEC and thermal stability observed. Additionally, statistical analyses were carried out and the correlation between parameters were evaluated. The overall results obtained would be a comprehensive evaluation on the effect of different acid modifications on TWBC that would become helpful for the judicious selection of an appropriate adsorbate to be used in applications of interest.

\section{Material and methods}

\subsection{Reagents, apparatus and equipment}

Analytical grade reagents, hydrochloric acid, nitric acid, sulfuric acid, sodium hydroxide pellets, sodium carbonate, sodium bicarbonate, sodium chloride, sodium acetate, ammonium acetate and ethanol were purchased from Sigma Aldrich (St. Louis, MO). Calibration standards for the atomic absorption spectrometer (AAS) were purchased from Inorganic Ventures (Christiansburg, VA). A Nabertherm GmbH, Germany muffle furnace equipped with a hardening box was used for the BC production. A KEM EBU-61020B automatic titrator, Kyoto Electronics Manufacturing Co. Ltd. (Japan) was used for Boehm titrations. A Metrohm 7025M (Switzerland) digital $\mathrm{pH}$ meter and a Thermo Scientific, (Waltham, MA) model 5 STAR electrical conductivity meter was used for $\mathrm{pH}$ and conductivity measurements. A Daihan Scientific, (Korea) water bath shaker was used for drying purposes. A Hitachi ZA3000 polarized Zeeman AAS (Japan) was utilized for the determination of CEC. For the functional group analysis, ABB MB300D Fourier transform infrared spectrophotometer (Switzerland) with attenuated total reflection (ATR) probe was incorporated. Total carbon (C), nitrogen $(\mathrm{N})$, hydrogen $(\mathrm{H})$ and oxygen $(\mathrm{O})$ content of BC samples were determined by dry combustion, using a CE - 440 (Exeter Analytical INC.) elemental analyzer. X-Ray Photoelectron Spectroscopy (XPS) analyses were performed using a Thermo Scientific K-Alpha $\left(\mathrm{K}_{\alpha}\right)$ XPS system equipped with a monochromatic X-ray source. Thermal stability of BC were determined using a thermogravimetric analyzer (TGA) (TA instruments, TGA Q 50). Scanning electron microscopic (SEM) images were obtained by a JEOL JSM-6500F FESEM instrument at $5 \mathrm{kV}$. Surface area analysis was carried out using a Micromeritics' TriStar II PLUS surface area and porosity system.

\subsection{Biochar production}

Tea-wastes were collected from three factories in Sri Lanka located in Nuwara eliya, Matale and Ambalangoda belonging to the subtropical highland, intermediate and tropical climate 
zones respectively. Dried biomass (length: $\sim 3.87 \mathrm{~cm}$ ) was packed well in an airtight hardening box to provide limited oxygen conditions and pyrolyzed using the muffle furnace under 300,500 and $700{ }^{\circ} \mathrm{C}$ (BC300, BC500 and BC700) according to the method described by Vithanage et al. with slight modifications. ${ }^{33}$ Briefly, the feedstock was heated to the desired temperature at a rate of $7{ }^{\circ} \mathrm{C} \min ^{-1}$ and held for $3 \mathrm{~h}$. The hardening box was allowed to cool inside the furnace overnight to avoid air oxidation. The produced BC were washed with deionized (DI) water until washings were clear and oven dried at $60{ }^{\circ} \mathrm{C}$ for $12 \mathrm{~h}$. Washed $\mathrm{BC}$ was ground and sieved to retain the 0.5-1 mm mesh fraction. Samples were kept in desiccators until use.

\subsection{Proximate and ultimate analysis}

Ash and moisture contents of $\mathrm{BC}$ were determined according to the American Society for Testing and Materials (ASTM) D286694 and D2867-17 methods respectively. ${ }^{34,35}$

The $\mathrm{C}, \mathrm{H}, \mathrm{O}$ and $\mathrm{N}$ compositions were used to determine polarity indexes. The $\mathrm{H} / \mathrm{C}$ atomic ratios were used as an indication of aromaticity. Furthermore, $\mathrm{O} / \mathrm{C}$ ratios and polarity indexes $((\mathrm{O}+\mathrm{N}) / \mathrm{C}$ ratios $)$ were calculated to evaluate polarities of the produced BCs. Thermogravimetric analyses were performed under $100 \mathrm{~mL} \mathrm{~min}{ }^{-1}$ nitrogen flow in the temperature range of $30-800{ }^{\circ} \mathrm{C}$. About $\sim 10 \mathrm{mg}$ of accurately weighed samples were used in each experiment. Statistical analyses were performed using R Core Team (2013). R: a language and environment for statistical computing. R Foundation for Statistical Computing, Vienna, Austria.

\subsection{Acid modifications of $\mathrm{BC}$}

Post modifications were carried out according to previously reported procedures with slight amendments. ${ }^{20,36,37}$ For the SM, a $100 \mathrm{~mL}$ portion of $10 \% \mathrm{H}_{2} \mathrm{SO}_{4}$ was added to $10 \mathrm{~g}$ of $\mathrm{BC}$ and stirred for $1 \mathrm{~h}$ at $60{ }^{\circ} \mathrm{C} .{ }^{36}$ The $\mathrm{NM}$ was performed by stirring $10 \mathrm{~mL}$ of $69 \% \mathrm{HNO}_{3}$ with $10 \mathrm{~g}$ of $\mathrm{BC}$ for $3 \mathrm{~h}$ at $60{ }^{\circ} \mathrm{C} .{ }^{20}$ For the $\mathrm{HM}$, a $100 \mathrm{~mL}$ portion of $5 \mathrm{M} \mathrm{HCl}$ was added to $10 \mathrm{~g}$ of $\mathrm{BC}$ and stirred for $24 \mathrm{~h}$ at $50{ }^{\circ} \mathrm{C} .{ }^{37}$ The modified $\mathrm{BC}$ was oven dried at $60{ }^{\circ} \mathrm{C}$ for $12 \mathrm{~h}$ after washing with DI until the washings became clear and neutral. The SM, NM and HM BCs will hereafter be abbreviated as SMBC, NMBC and HMBC respectively.

\subsection{Surface morphology and functionality}

To obtain SEM images, samples were coated on a carbon stub attached to a carbon tape and then was mounted onto a sample holder for SEM analysis. Surface functionality was evaluated by obtaining an ATR-FTIR spectra of the dry BC within a range of $4000-500 \mathrm{~cm}^{-1}$ with $4 \mathrm{~cm}^{-1}$ resolution through 64 scans.

The monochromatic X-ray source of the XPS analyzer was set to $1486.6 \mathrm{eV}$, corresponding to the $\mathrm{Al} \mathrm{K}_{\alpha}$ line, with a spot size of $400 \mu^{2}$. Photoelectrons were collected from a takeoff angle of $90^{\circ}$ relative to the overall sample's fractal particle surface. Measurements were done in the constant analyzer energy mode. The survey spectra were taken at a pass energy of $200 \mathrm{eV}$, while the high resolution core level spectra were taken at a $40 \mathrm{eV}$ pass energy.

\subsection{Measurements of EC, $\mathrm{pH}$ and PZC}

Biochar $\mathrm{pH}$ and EC measurements were carried out according to the method described elsewhere with slight modifications. ${ }^{33} \mathrm{~A}$ $1: 10(\mathrm{w} / \mathrm{v})$ BC: water suspension ratio was equilibrated at $100 \mathrm{rpm}$ in a mechanical shaker at room temperature for $4 \mathrm{~h}$ prior to measurements. Nitrogen purged DI was used for experiments in order to eliminate dissolved $\mathrm{CO}_{2}$ and the data were corrected with respect to a simultaneous blank run carried out using DI water.

The PZC of BC was determined by the $\mathrm{pH}$ drift method described in Ferro-Garcia et al. Briefly, $60 \mathrm{mg}$ samples of BC were shaken with $20 \mathrm{~mL}$ portions of $0.01 \mathrm{M} \mathrm{NaCl}$ solutions preadjusted to $\mathrm{pH} 2,4,6,8$, and 10 with $0.1 \mathrm{M} \mathrm{HCl}$ or $\mathrm{NaOH}$ for $24 \mathrm{~h}$ at $25{ }^{\circ} \mathrm{C}$ and the final $\mathrm{pH}$ was measured. Sodium chloride solutions were purged with $\mathrm{N}_{2}$ upon preparation to prevent the dissolution of $\mathrm{CO}_{2}$.

\subsection{Surface acidity and basicity}

Acidic and basic functional group distribution on the BC surface was determined by performing Boehm titrations. To evaluate surface acidity, an accurately weighed aliquot of BC $(\sim 0.5000 \mathrm{~g})$ was shaken either with $50 \mathrm{~mL}$ portions of $0.05 \mathrm{M}$ $\mathrm{NaHCO}_{3}, \mathrm{Na}_{2} \mathrm{CO}_{3}$, or $\mathrm{NaOH}$ for $12 \mathrm{~h}$. After equilibration, the suspension was filtered and titrated with $0.05 \mathrm{M} \mathrm{HCl}$ using the autotitrator. The Boehm titration assumes that $\mathrm{NaHCO}_{3}$ neutralizes stronger acids (mainly carboxylic), $\mathrm{Na}_{2} \mathrm{CO}_{3}$ neutralizes stronger and moderate acids (mainly low $\mathrm{p} K_{\mathrm{a}}$ phenols and hydrolysis products of lactones), and $\mathrm{NaOH}$ neutralizes all acidic moieties including weak acids (mainly high $\mathrm{p} K_{\mathrm{a}}$ phenols). Surface basicity was determined by shaking BC with $0.05 \mathrm{M} \mathrm{HCl}$ and subsequently titrating with $0.05 \mathrm{M} \mathrm{NaOH}$ in a similar manner. Biochar samples were pretreated with $\mathrm{NaOH}$ to remove solubilizable acidic species and humic substances, and afterwards with $\mathrm{HCl}$ to remove solubilizable basic species and to protonate O-SFGs as described by Tsechansky et al. ${ }^{38}$

\subsection{Cation exchange capacity}

The ammonium acetate compulsory displacement method described by Sumner and Miller was utilized for CEC determinations with slight modifications. ${ }^{39}$

Concisely, accurately weighed aliquots of BC $(\sim 1.000 \mathrm{~g})$ were shaken in $\mathrm{pH}$ adjusted sodium acetate $(0.5 \mathrm{M}$ solutions adjusted to $\mathrm{pH}=3,7$ and $10 \mathrm{using} \mathrm{HCl}$ and $\mathrm{NaOH}$ ) at $200 \mathrm{rpm}$ for $1 \mathrm{~h}$ and filtered under vacuum using a Whatman 42 filter paper. The BC was washed five times with $30 \mathrm{~mL}$ portions of the same acetate solution to ensure the saturation with sodium ions. Biochar samples were then washed three times with $20 \mathrm{~mL}$ of ethanol to remove excess of sodium ions and the washings were discarded. Exchanged sodium ions were displaced with $30 \mathrm{~mL}$ of $0.5 \mathrm{M}$ ammonium acetate five times and the collected washings were quantitatively diluted and measured using the AAS.

\section{Results and discussion}

\subsection{Pyrolysis temperature selection}

Biochar production entails the conversion of hemicellulose, cellulose and lignin fractions of the biomass into disordered 
recalcitrant organic carbon which has high stability and a life span of more than a century, depending on production conditions. ${ }^{40-42}$ The devolatilization and carbonization of hemicellulose and cellulose begins at a temperatures starting from 180-240 ${ }^{\circ} \mathrm{C}$ and $230-310{ }^{\circ} \mathrm{C}$ respectively. Decomposition of lignin starts from temperatures as low as $160{ }^{\circ} \mathrm{C}$ and continues slowly until up to $900{ }^{\circ} \mathrm{C} .{ }^{11}$ The lower margin of $\mathrm{BC}$ production temperature by slow pyrolysis can be considered as $\sim 300{ }^{\circ} \mathrm{C}$ as only a limited decomposition of hemicellulose is observed at lesser temperatures. ${ }^{43}$

Aromatization reactions predominate at temperatures beyond $400{ }^{\circ} \mathrm{C}$ and the fraction of aromatic domains are rapidly increased at elevated temperatures. ${ }^{44}$ When the temperature exceeds $700{ }^{\circ} \mathrm{C}$, the carbon structure becomes more ordered and graphitized increasing the size of aromatic domains. ${ }^{45,46}$ Turbostratic structures have been observed at temperatures as high as $800{ }^{\circ} \mathrm{C}$ and the structure becomes graphitic with order in the third dimension when the temperature exceeds $1500{ }^{\circ} \mathrm{C}$, significantly reducing the stability and porosity of BC. ${ }^{46}$ Hence, $\mathrm{BC}$ for the presented study were prepared at the two borderline temperatures, 300 and $700{ }^{\circ} \mathrm{C}$ and at an average temperature of $500{ }^{\circ} \mathrm{C}$.

\subsection{Surface morphology and functionality}

3.2.1. Surface morphology. The amorphous and heterogeneous structure of TWBCs were confirmed by SEM images. Variation of surface morphology with pyrolysis temperature is well documented in literature through SEM image based observations. ${ }^{\mathbf{2 6 4 7 - 4 9}}$ It has been reported that the orderliness of the graphitized structure and the degree of aromaticity increased with the pyrolysis temperature due to the volatilization of oxygen and hydrogen from the low aromaticity lignocellulosic compounds and lignin causing the remaining carbon to form new aromatic bonds. ${ }^{47}$ Release of volatile compounds create a considerable internal pressure causing the coalescence of the smaller pores, forming more enlarged pores. ${ }^{\mathbf{2 6 , 4 8 , 4 9}}$ Further, SEM images of BC produced under high temperatures have provided indirect evidence of the release of volatile compounds during pyrolysis. Obtained SEM images shown in Fig. 1A-C support the above phenomena. Even though pore wall destruction can be seen upon all three acid modifications, sulphuric acid treatment showed maximum effect due to the surplus water vapor released through the dehydration of sulphuric acid than the nitric and hydrochloric acids according to SEM images (Fig. 1D-F). ${ }^{20}$ Surface areas were increased upon increasing pyrolysis temperature and BC700 showed considerable increase upon acid modifications (Table S1, ESI $\dagger$ ).

3.2.2. FTIR analysis. The variation of FTIR spectra of TWBC with pyrolysis temperature and different acid modifications are shown in Fig. 2. The SFGs of BC are derived by the decomposition of organic components in feedstock such as lignin, cellulose and hemicellulose. Three major bands of FTIR spectra were identified at wavenumbers $3200-3500 \mathrm{~cm}^{-1}$ and $2820-$ $2980 \mathrm{~cm}^{-1}$ corresponding to $-\mathrm{OH}$ stretching of alcohol/ carboxylic and $-\mathrm{CH}_{2}$ stretching of polar groups respectively. The peak at $885-750 \mathrm{~cm}^{-1}$ is representative of out of plane isolated and substituted $\mathrm{C}-\mathrm{H}$ in the aromatic structure and also of carbonates present. The XPS data (Section 3.2.3) provides validation for the presence of carbonates in the biochar structure. Overall intensity of FTIR spectra was decreased with increasing pyrolysis temperature. The diminution of the alcohol/carboxylic - $\mathrm{OH}$ stretching can be attributed to dehydration whereas the disappearance of $-\mathrm{CH}_{2}$ aliphatic bands indicated a decrease in polar functional groups.

An intense peak assigned to carbonyl stretching was observed around $1690-1720 \mathrm{~cm}^{-1}$ in NMBC and SMBC due to the introduction of carboxylic acids. A peak around 1200$1250 \mathrm{~cm}^{-1}$ in HMBC representing $\mathrm{C}-\mathrm{O}$ stretching bond can be due to the formation of singly bonded $\mathrm{C}-\mathrm{O}$ moieties in lactones and phenols. The appearance of a band at $874 \mathrm{~cm}^{-1}$ in BC500 and $\mathrm{BC} 700$ indicated the formation of larger aromatic domains. ${ }^{32,49,50}$ A similar observation was reported by Ahmed et al..$^{50}$

3.2.3. XPS analysis. Low resolution XPS spectra for BC300, BC500, BC700 and acid modified BC (SM, HM, NM) are given in ESI (Fig. S2†). The survey spectrum shows changes in the elemental composition upon acid treatment. Traces of $\mathrm{Mg}, \mathrm{Mn}$ and $\mathrm{Si}$ are detected in $\mathrm{BC}$ precursors and $\mathrm{Mg}$ and $\mathrm{Mn}$ are seen to disappear with acid treatment. This could be due to the dissolution of $\mathrm{Mg}$ and $\mathrm{Mn}$ oxides upon acid treatment. However, oxides of Si are highly stable in these acids and not removed during acid modification.

The O1s high resolution envelope for BC300, BC500 and BC700 and BC after acid modification was resolved into five signals. The XPS spectra for raw and modified BC300, BC500 and BC700 are given in Fig. 3, while the associated data of the raw and modified BC are given in the ESI (Tables S3-S6†).

The highest energy contribution in 535.1-533.7 eV range can be attributed to- $\mathrm{NO}_{2},-\mathrm{O}-\mathrm{NO}_{2}$ functionalities. A binding energy (BE) range of 533.7-532.88 eV can be attributed to $\mathrm{O}=\mathrm{C}-\mathrm{OH}(\mathrm{R})$ functionalities. Carbonate $\left(\mathrm{CO}_{3}{ }^{2-}\right)$ type functionalities occur at 532.8-531.99 eV BE range. $\mathrm{O}-\mathrm{C}-$ and $\mathrm{O}=\mathrm{C}$ - functionalities exist in 531.74-531.03 eV and 530.26-530.75 $\mathrm{BE}$ range respectively. ${ }^{51}$ When increasing the pyrolysis temperature from $300{ }^{\circ} \mathrm{C}$ to $500{ }^{\circ} \mathrm{C}, \mathrm{O}=\mathrm{C}-\mathrm{OH}(\mathrm{R})$ atomic percentage have increased slightly. This could be due to an increase in carbonates with increasing pyrolysis temperature and partial overlapping of $\mathrm{O}=\mathrm{C}-\mathrm{OH}(\mathrm{R})$ with $\mathrm{CO}_{3}{ }^{2-}$ spectrum leading to a net increase in atomic percentages. But however, with further increase in temperature the atomic percentage drops drastically. This can be attributed to the loss in $\mathrm{O}=\mathrm{C}-\mathrm{OH}(\mathrm{R})$ functionalities because of decarboxylation at high temperatures.

The C1s high resolution envelop was curve resolved into four signals which are listed and assigned in Table S $3 \dagger$ and Fig. 3 respectively. The highest energy contribution can be attributed to $\mathrm{O}=\mathrm{C}-\mathrm{OH}(\mathrm{R}), \mathrm{CO}_{3}{ }^{2-}$ in $\mathrm{BE}$ range of $289.21-288.53 \mathrm{eV} . \mathrm{C}=\mathrm{O} /-$ $\mathrm{C}-\mathrm{N}$, and $\mathrm{O}-\mathrm{C}-$ occurs in $287.89-287.28 \mathrm{eV}$ and 286.13$284.92 \mathrm{eV}$ (BE) range respectively. $\mathrm{C}-\mathrm{C}$ and $\mathrm{C}-\mathrm{H}$ co-occur in $\mathrm{BE}$ 284.46-284.28 eV range. ${ }^{51}$ Similar to $\mathrm{O} 1 \mathrm{~s} \mathrm{O}=\mathrm{C}-\mathrm{OH}(\mathrm{R}), \mathrm{CO}_{3}{ }^{2-}$ atomic percentage increased with increasing temperature and dropped with further increment. Individual contribution from $\mathrm{CO}_{3}{ }^{2-}$ and $\mathrm{O}=\mathrm{C}-\mathrm{OH}(\mathrm{R})$ cannot be estimated as they co-exist. $\mathrm{O}-\mathrm{C}-$ has been decreased with increasing carbonization 


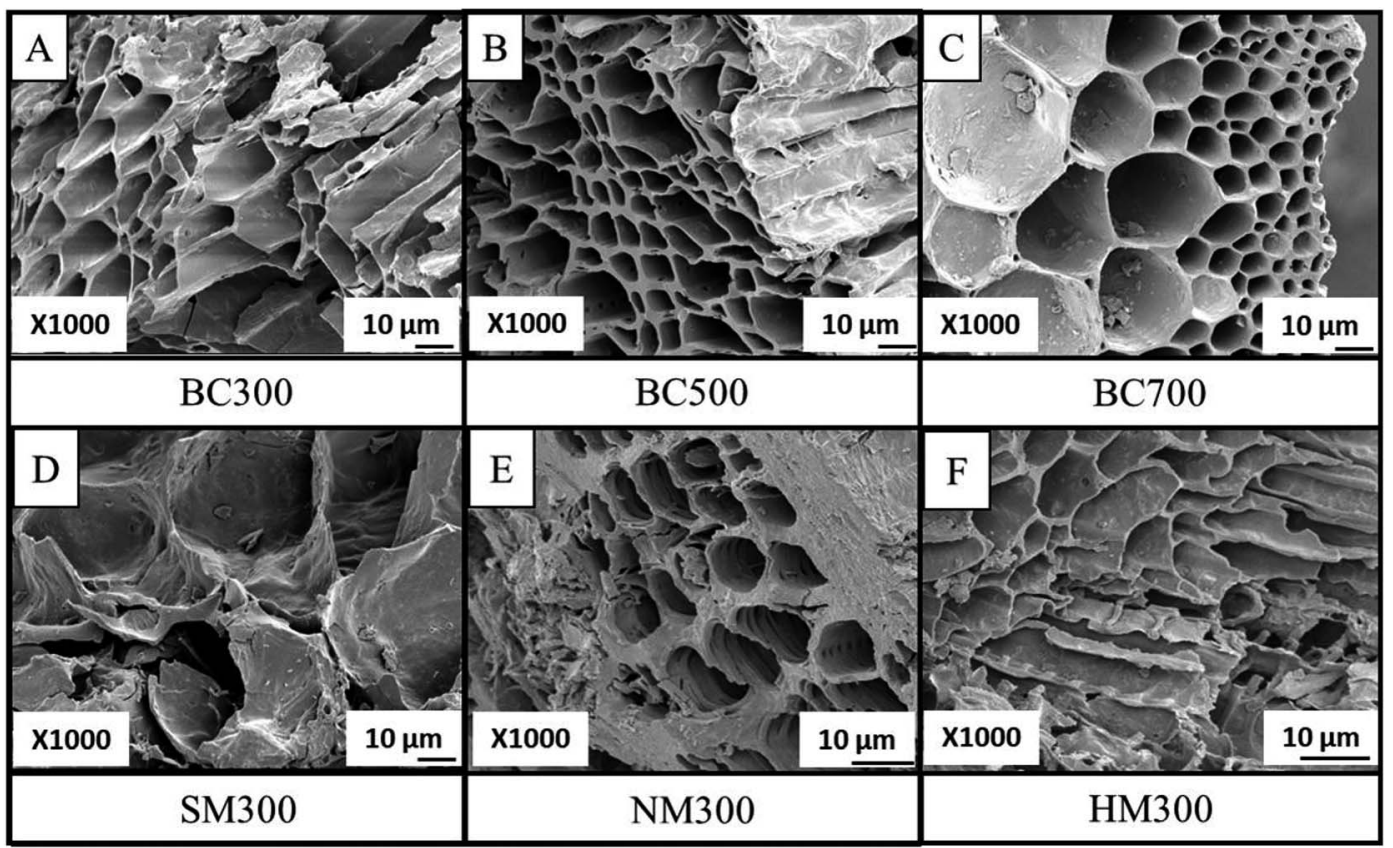

Fig. 1 SEM images of raw BCs (A) BC300, (B) BC500 and (C) BC700, and modified BCs (D) SM300, (E) NM300 and (F) HM300.

temperature. Aromaticity of the char has been increased with the carbonization temperature.

The N1s high resolution envelop has been curve resolved into five peaks. The highest $\mathrm{BE}$ can be attributed to $-\mathrm{NO}_{2},-\mathrm{O}-$ $\mathrm{NO}_{2}$ (496.26-494.08 eV BE). Quaternary-N occurs around BE 403.51-401.54 eV range. The pyrrolic-N, pyridonic- $\mathrm{N}$ and pyridinic-N occurs in the 400.81-400.29 eV, $399.91-399.37 \mathrm{eV}$ and 398.62-397.96 eV range respectively. ${ }^{52}$ Quaternary-N shows no significant change with increasing pyrolysis temperature. However, the pyrrolic-N atomic percentage has been decreased with increasing pyrolysis temperature possibly due to deamination. Subsequently, the aromatic pyridonic- $\mathrm{N}$ and pyridinic- $\mathrm{N}$ has been increased which further suggests that the aromaticity of the $\mathrm{BC}$ has been increased with increasing pyrolysis temperature.
Upon nitration, the nitro and nitroxy atomic percentages (in O1s and N1s) have been increased in NM300, NM500 and NM700 compared to their precursor BCs; BC300, BC500 and $\mathrm{BC} 700$ respectively, and with increasing pyrolysis temperature they further increase (Fig. 3). This is due to the availability of aromatic rings to drive the nitration reaction. $\mathrm{C} 1 \mathrm{~s} \mathrm{O}=\mathrm{C}-\mathrm{OH}(\mathrm{R})$, $\mathrm{CO}_{3}{ }^{2-}$ atomic percentages have been increased and this can be attributed to the increase in $\mathrm{O}=\mathrm{C}-\mathrm{OH}(\mathrm{R})$ moieties upon NM.

Hydrochloric acid modification resulted in low surface acidic functionalities $(1.74-3.63 \%$ in $\mathrm{O} 1 \mathrm{~s}$ and $2.8-3.25 \%$ in $\mathrm{C} 1 \mathrm{~s})$ compared to the precursor BCs. Also, the carbonate atomic percentages have been decreased compared to the precursor $\mathrm{BCs}$, as $\mathrm{HCl}$ can titrate the carbonates of $\mathrm{Mg}, \mathrm{Ca}$ and $\mathrm{Na}$ etc.

Sulfuric acid modification has failed to increase any carboxylic acidic functionalities compared to its precursor BC
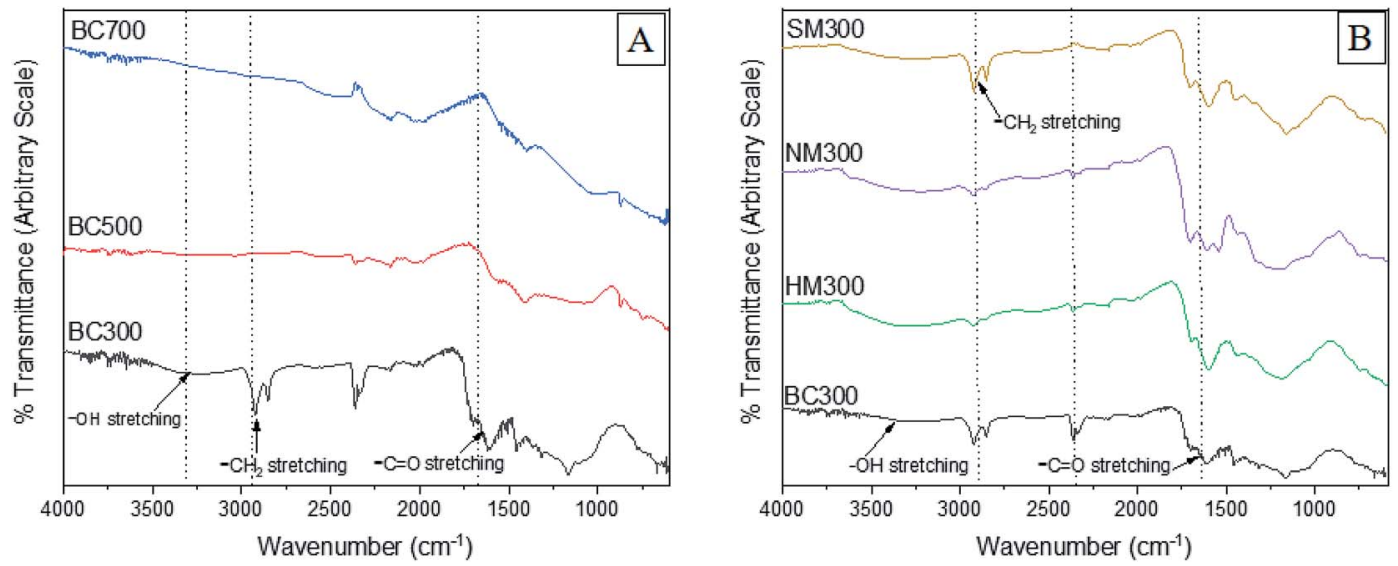

Fig. 2 FTIR spectra of (A) raw BCs and (B) modified BCs. 


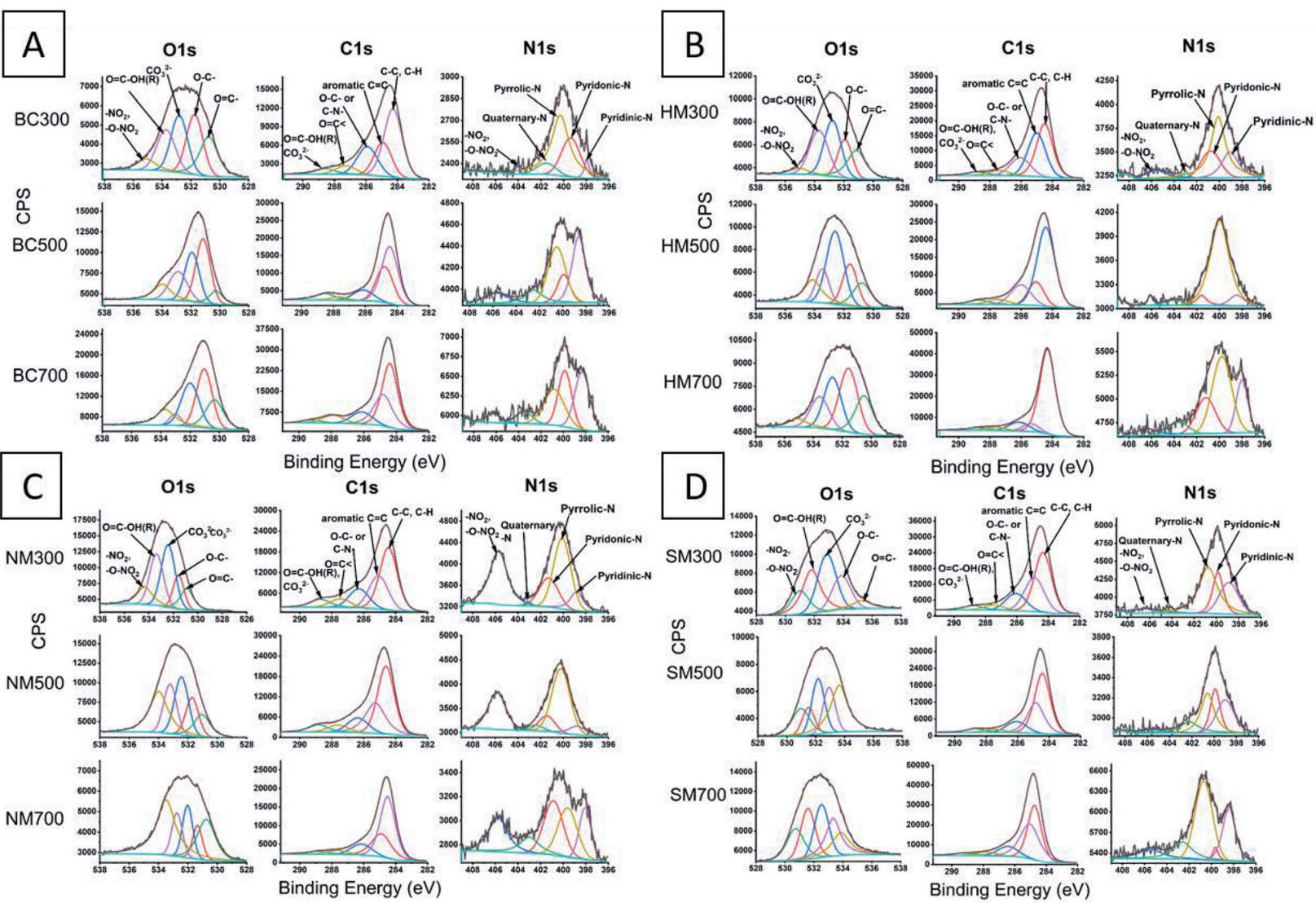

Fig. 3 High resolution (HR) O1s, C1s and N1s XPS spectra for (A) raw BC, (B) HMBC, (C) NMBC and (D) SMBC.

as, it can catalyze the decarboxylation of existing carboxylic groups. ${ }^{24}$ However, several new peaks appeared in S2p for the SM700. The S2p high resolution envelope was deconvoluted to

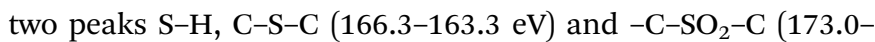
$165.9 \mathrm{eV}$ ) (Fig. S7†). S2p only appeared in SM700. This could be due to high aromaticity present in SM700 and sulfonation being readily possible due to the high aromaticity. Presence of C-S-C peak suggests that the $\mathrm{BC}$ has been slightly cross linked with sulfur. Possible key reaction mechanisms upon the three acid modifications are shown in Fig. 4.

\subsection{Surface acidity and basicity}

A significant diminution of total surface acidity was observed with increasing pyrolysis temperature due to volatilization and degradation (Fig. 5A). Biochar produced at $500{ }^{\circ} \mathrm{C}$ contained 1.7 times more O-SFGs than $\mathrm{BC} 700$ and $\mathrm{BC} 300$ contained 2.7 times more O-SFGs than BC500. High $\mathrm{p} K_{\mathrm{a}}$ phenols had the highest contribution to surface acidity in all three raw BCs (73-82\%). Carboxylic moieties exhibited a $17 \%$ contribution to the total surface acidity of BC300 which was reduced to $8 \%$ and $5 \%$ in BC500 and BC700 respectively. The highest percentage of lactonic and low $\mathrm{p} K_{\mathrm{a}}$ phenolic SFGs were observed in BC700 which was $21 \%$ while it was reduced to $5 \%$ in BC500 and to less than $1 \%$ in BC300. In contrast to surface acidity, increased surface basicities were observed as the pyrolysis temperature was increased (Fig. 5A). The production of basic moieties such as pyrones and chromens at elevated pyrolysis temperatures could be the reason for this observation. ${ }^{53}$

Boehm titrations were also performed for acid modified BC300 and BC500. Significant enhancements of total surface acidity were observed upon all three acid modifications (Fig. 5B and C). For both BC300 and BC500, the highest enrichment of total surface acidity was obtained upon NM ( 1.6-1.8 times) which was solely due to the introduction of carboxylic moieties. It has been reported that both the introduction of new O-SFGs via oxidative ring opening of aromatic rings and the oxidation of existing O-SFGs can occur during NM (Fig. 4A). ${ }^{20,22}$ An increment in lactonic groups was observed upon HM in both HM300 and HM500 as hydrochloric acid is capable of reducing O-SFGs. ${ }^{24}$ The reaction mechanism involved in the introduction of single-bonded O-SFGs is included in Fig. 4C. Among the three acid treatment methods, the lowest surface acidity enhancement was caused by the SM. The observed decrement in carboxylic moieties upon SM can be attributed to decarboxylation reactions (Fig. 4B).

The increment of specific surface area and the resultant exposure of unavailable SFGs caused by the demineralization effect during an acid treatment can also contribute to the enrichment of surface acidity. ${ }^{54}$ As the basic moieties on carbonaceous surfaces are already being neutralized by acid treatment, basicity evaluations of modified BC were not evaluated. 

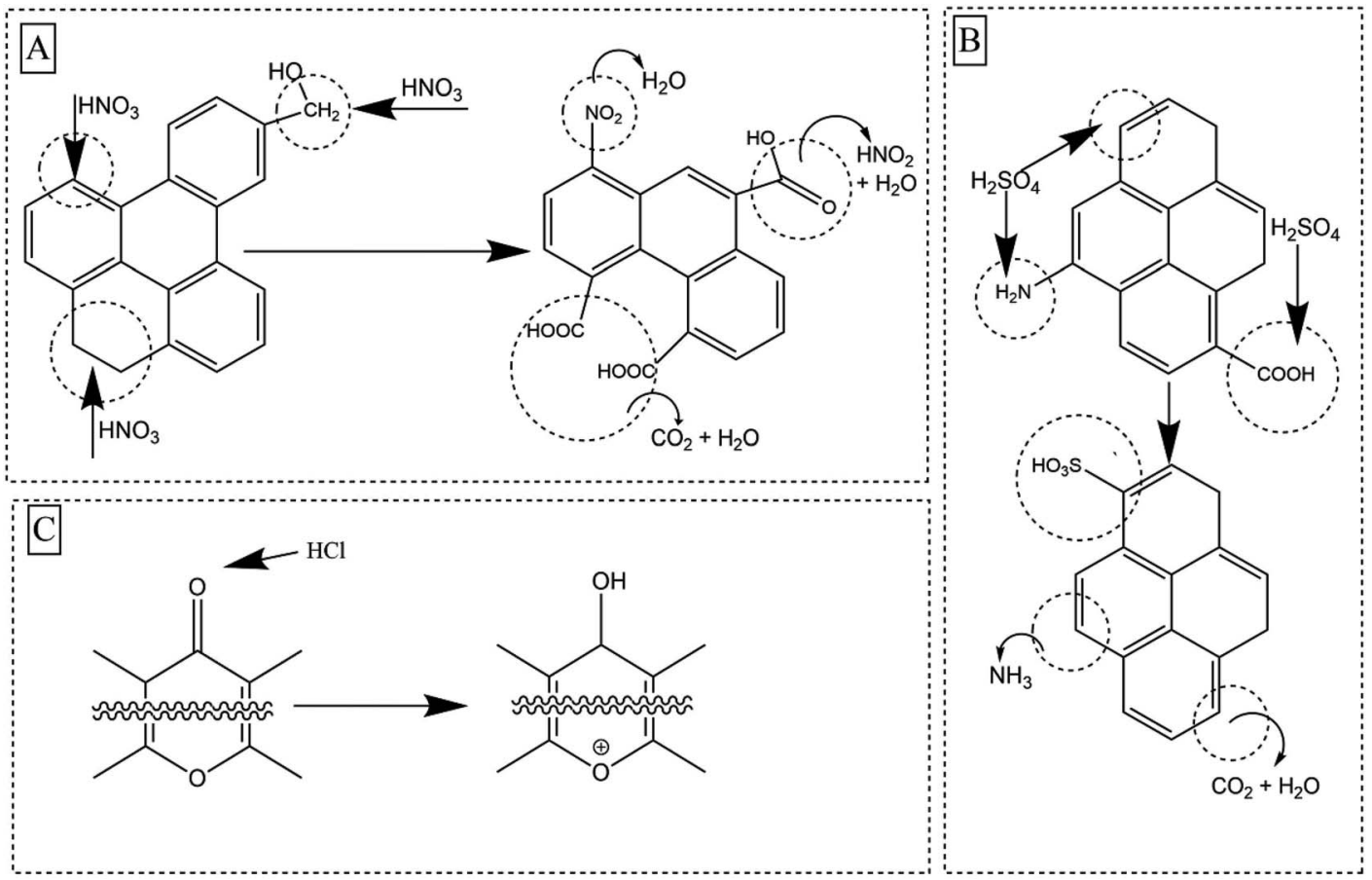

Fig. 4 Proposed reaction mechanisms involved in (A) nitric, (B) sulfuric and (C) hydrochloric modification.

\subsection{Proximate and ultimate analysis}

3.4.1. Yield, ash and moisture content. Maximum decomposition of biomass during pyrolysis occurs from $\sim 200$ $400{ }^{\circ} \mathrm{C} .{ }^{55}$ The $\mathrm{BC}$ yield sharply decreased when the pyrolysis temperature was increased from 300 to $500{ }^{\circ} \mathrm{C}$ and only slightly decreased when it was further increased to $700{ }^{\circ} \mathrm{C}$, indicating that the majority of the volatile fraction has been removed at lower temperatures (Table 1).

The ash content increased from $6.3 \%$ to $11.4 \%$ when the pyrolysis temperature was increased from 300 to $500{ }^{\circ} \mathrm{C}$ and slightly decreased to $9.3 \%$ when the temperature was further increased to $700{ }^{\circ} \mathrm{C}$. Zhao et al. also reported a similar observation for apple tree branch $\mathrm{BC} .^{55}$ The possible reason for this observation may be the increased amount of inorganic materials in $\mathrm{BC} 500$ compared to $\mathrm{BC} 300$ and the partial devolatilization of produced inorganic constituents in BC700. Decreased ash contents were observed upon all three acid modifications suggesting the removal of trapped inorganic constituents (Table 1).

The C content of $\mathrm{BC} 300$ which was $58 \%$ was increased to $71 \%$ for BC700. In contrast, the oxygen content decreased from $34 \%$ to $24 \%$ when going from BC300 to BC700. These two facts indicated an increase in carbonization and a decrease in polarity when the temperature was increased.

3.4.2. Elemental analysis. The molar $\mathrm{O} / \mathrm{C}$ ratios and the $(\mathrm{O}$ $+\mathrm{N}$ )/C ratios were observed to decrease with increasing pyrolysis temperature stipulating that $\mathrm{BC}$ produced at higher temperatures were hydrophobic. This was as a result of the volatilization of polar functional groups. At higher pyrolysis temperatures, the molar $\mathrm{H} / \mathrm{C}$ ratios were seen to decrease as well indicating that $\mathrm{BC}$ produced at elevated temperatures possessed a highly aromatic structure. ${ }^{32}$ A similar pattern was observed in acid modified BCs. Elemental compositions as well as $\mathrm{H} / \mathrm{C}$ and $(\mathrm{O}+$ $\mathrm{N})$ /C ratios of raw and modified BC are shown in Table 1.

3.4.3. Thermo gravimetric analysis (TGA). The TGA analysis was used to investigate changes in the thermal stability of the BC after the acid treatment process. Fig. S8 $\dagger$ shows the TGA curves for raw BC, SMBC, HMBC and NMBC. The BC 300 possessed poor thermal stability, which could be due to the decarboxylation and, decomposition of remaining lignocellulose material. ${ }^{56}$ Biochar produced at $500{ }^{\circ} \mathrm{C}$ and $\mathrm{BC} 700$ are fairly stable in the TGA experiment temperature, as they were produced from high temperature pyrolysis. However, $\sim 15 \%$ of the weight loss can be attributed to the loss of volatile organic compounds (VOC) and decarboxylation of BC. ${ }^{56}$ Sulfuric acid modification decreased the stability of BC500 and BC700. Consequently, the stability of BC300 has been increased. This weight loss of SM500 and SM700 could be possibly due to loss of VOCs and de-sulfonation. The SM500 and SM700 may have more degree of sulfonation as their precursor BC's (BC500 and BC700) are more aromatic. Both HM and NM markedly decrease the graphitic and aromatic carbons in BC300 and BC500 and increases the oxygenated and nitrogenated functionalities. ${ }^{24}$ It leads to poor thermal stability of HM/ NM 300 and 500. The aromaticity of BC700 is not susceptible or not changed significantly upon NM and HM which leads to higher thermal stability.

\section{5. $\quad \mathrm{pH}$ and electrical conductivity measurements}

Organic matter, inorganic constituents and SFGs can be considered as the main factors affecting the $\mathrm{pH}$ of $\mathrm{BC} .{ }^{57}$ Low temperature produced $\mathrm{BC}$ is commonly expected to have acidic $\mathrm{pH}$ as a result of the formation of O-SFGs and various other 
Table 1 Proximate and ultimate analysis of raw and three acid modified TWBCs produced under three different pyrolysis temperatures ${ }^{a}$

\begin{tabular}{|c|c|c|c|c|c|c|c|}
\hline \multirow[b]{2}{*}{ Sample } & \multicolumn{7}{|c|}{ Proximate analysis } \\
\hline & Yield (\%) & \multicolumn{2}{|l|}{ Moisture (\%) } & Ash (\%) & $\mathrm{pH}$ & $\mathrm{pH}_{\mathrm{PZC}}$ & $\mathrm{EC}\left(\mu \mathrm{S} \mathrm{cm}^{-1}\right)$ \\
\hline BC300 & $52.17 \pm 1.28$ & $6.33 \pm 0.57$ & \multicolumn{2}{|r|}{$6.15 \pm 0.22$} & $7.16 \pm 0.01$ & 6.31 & $12025.00 \pm 0.07$ \\
\hline BC500 & $32.58 \pm 2.17$ & $9.70 \pm 0.60$ & \multicolumn{2}{|r|}{$11.40 \pm 0.25$} & $7.04 \pm 0.02$ & 9.16 & $2514.50 \pm 0.07$ \\
\hline BC700 & \multirow[t]{10}{*}{$28.17 \pm 1.89$} & $7.97 \pm 0.04$ & \multicolumn{2}{|r|}{$9.26 \pm 0.37$} & $10.09 \pm 0.05$ & 7.51 & $1984.00 \pm 1.41$ \\
\hline NM300 & & & \multicolumn{2}{|r|}{$2.80 \pm 0.28$} & $2.40 \pm 0.01$ & 4.67 & $1457.65 \pm 0.70$ \\
\hline NM500 & & & \multicolumn{2}{|r|}{$3.10 \pm 0.35$} & $2.44 \pm 0.00$ & 4.96 & $1264.50 \pm 0.70$ \\
\hline NM700 & & & \multicolumn{2}{|r|}{$2.50 \pm 0.71$} & $2.57 \pm 0.01$ & 5.55 & $1224.50 \pm 1.41$ \\
\hline SM300 & & & \multicolumn{2}{|r|}{$4.00 \pm 0.35$} & $3.40 \pm 0.01$ & 5.35 & $1187.00 \pm 1.41$ \\
\hline SM500 & & & \multicolumn{2}{|r|}{$3.50 \pm 0.79$} & $2.35 \pm 0.04$ & 5.45 & $1607.50 \pm 0.07$ \\
\hline SM700 & & & \multicolumn{2}{|r|}{$6.50 \pm 1.01$} & $4.16 \pm 0.00$ & 6.95 & $663.00 \pm 2.82$ \\
\hline НМ300 & & & \multicolumn{2}{|r|}{$3.10 \pm 0.18$} & $2.55 \pm 0.00$ & 5.67 & $482.50 \pm 0.70$ \\
\hline HM500 & & & \multicolumn{2}{|r|}{$3.80 \pm 0.53$} & $2.65 \pm 0.00$ & 5.60 & $418.00 \pm 1.41$ \\
\hline HM700 & & & \multicolumn{2}{|r|}{$7.00 \pm 0.83$} & $2.49 \pm 0.01$ & 2.65 & $1191.00 \pm 1.41$ \\
\hline & Ultimate & & & & & & \\
\hline & $\mathrm{C}(\%)$ & H (\%) & $\mathrm{N}(\%)$ & $\mathrm{O}(\%)$ & Molar H/C & $\begin{array}{l}\text { Molar } \\
\text { O/C }\end{array}$ & $\begin{array}{l}\text { Molar }(\mathrm{O}+ \\
\mathrm{N}) / \mathrm{C}\end{array}$ \\
\hline Tea-waste & 47.80 & 5.12 & 3.75 & 42.17 & 1.29 & 0.68 & 0.75 \\
\hline BC300 & 57.80 & 4.42 & 3.66 & 34.12 & 0.92 & 0.44 & 0.50 \\
\hline BC500 & 69.66 & 2.96 & 2.55 & 24.82 & 0.51 & 0.27 & 0.30 \\
\hline BC700 & 71.03 & 2.11 & 3.12 & 23.74 & 0.36 & 0.25 & 0.29 \\
\hline NM300 & 57.03 & 4.47 & 5.51 & 32.99 & 0.94 & 0.43 & 0.52 \\
\hline NM500 & 56.57 & 4.39 & 5.26 & 33.78 & 0.93 & 0.45 & 0.53 \\
\hline NM700 & 71.30 & 2.21 & 3.80 & 22.69 & 0.37 & 0.24 & 0.28 \\
\hline SM300 & 60.79 & 4.69 & 3.87 & 30.65 & 0.93 & 0.38 & 0.43 \\
\hline SM500 & 61.27 & 4.55 & 3.92 & 30.26 & 0.89 & 0.37 & 0.43 \\
\hline SM700 & 71.37 & 2.14 & 3.04 & 23.45 & 0.36 & 0.25 & 0.28 \\
\hline HМ300 & 63.15 & 4.75 & 3.92 & 28.18 & 0.90 & 0.34 & 0.39 \\
\hline HM500 & 63.35 & 4.17 & 3.88 & 28.07 & 0.79 & 0.33 & 0.39 \\
\hline HM700 & 74.02 & 2.22 & 3.15 & 20.62 & 0.36 & 0.21 & 0.25 \\
\hline
\end{tabular}

acidic organic substances while high temperature BC generally exhibits alkaline $\mathrm{pH}$ due to the higher rates of carbonization of organic matter and the removal of SFGs. ${ }^{43}$ However in this study, both BC300 and BC500 showed neutral pH while BC700 showed mildly basic $\mathrm{pH}$ (Table 1). It is interesting to note the insignificant difference in $\mathrm{pH}$ of $\mathrm{BC} 300$ and $\mathrm{BC} 500$ despite the variation of their ash content. Though BC300 contained a significantly higher amount of acidic O-SFGs as compared to $\mathrm{BC} 500$, their contribution to $\mathrm{pH}$ can be assumed to be negligible because the total surface acidity was mainly contributed to by weak, high $\mathrm{p} K_{\mathrm{a}}$ phenols. It is also important to note the increment of basic SFGs and the decrement of acidic SFGs when the pyrolysis temperature was increased from 300 to $700{ }^{\circ} \mathrm{C}$. Though the exact phenomenon for the $\mathrm{pH}$ variation with pyrolysis temperature is unclear, it can be concluded that the SFGs play a dominating role on the $\mathrm{BC} \mathrm{pH}$.

Dissolution of ash content in $\mathrm{BC}$ is a key factor contributing to the EC. ${ }^{58}$ Among the three raw BC, the highest EC was observed by BC500 (Table 1) and the high ash content justifies this observation. The release of the soluble organics such as humic substances in BC500 could also be a factor contributing to the elevated EC.
As a whole, ECs were reduced upon acid modifications (Table 1). The highest diminution in EC was observed in HMBC whereas the lowest diminution was observed in NMBC. The EC of NMBC300 was slightly higher than that of BC300 suggesting the contribution of a high amount of added carboxylic groups which are capable of providing protons to the medium.

\subsection{Point of zero charge}

For raw BC, only BC300 showed an acidic PZC and the highest PZC was observed in BC500. Low PZC of BC300 can be as a result of a high content of acidic O-SFGs and the highly basic PZC of BC500 can be due to the high ash content. The observed PZC values were decreased upon acid modifications (Table 1). This can be explained by the demineralization effect that lowers the ash content as well as the appearance of new acidic O-SFGs.

\subsection{Cation exchange capacity}

Cation exchange capacities of modified and raw $\mathrm{BC}$ produced at 300 and $500{ }^{\circ} \mathrm{C}$ were evaluated at $\mathrm{pH}$ of 3,7 and 10 . Significantly higher CECs were observed in BC300 when compared to BC500. As confirmed by Boehm titrations, BC300 contained 
a significantly higher amount of O-SFGs which can act as favorable binding sites.

Results also showed that the CEC for a given $\mathrm{BC}$ is increased with the $\mathrm{pH}$ of the medium (Fig. 5D and E). For raw and NMBC, sharper enhancements $(3.5 \times$ and $4.5 \times$ respectively) of CEC were observed when the $\mathrm{pH}$ was increased from 3 to 7 whereas comparatively slighter enhancements were observed upon $\mathrm{pH}$ increment from 7 to $10(2.1 \times$ and $1.1 \times$ respectively).

The contribution of carboxylic and lactonic groups can be a possible reason for this observation. Since the $\mathrm{p} K_{\mathrm{a}}$ values of carboxylic and lactonic groups generally range from 2-4 and 4-7 respectively, it can be concluded that the majority of these O-SFGs can be in their deprotonated form when the $\mathrm{pH}$ reaches 7 , acting as excellent binding sites for cations via electrostatic interactions. The slight increase in CEC when the $\mathrm{pH}$ was increased from 7 to 10 may be due to the deprotonation of phenolic groups which have lesser contribution to CEC. ${ }^{59}$

Acid modifications have generally increased the CEC. Introduction of binding sites, pore widening and opening of unavailable pores can be considered as the major reasons responsible for this observation. ${ }^{3,20}$ The enhancement of CEC at acidic $\mathrm{pH}$ can be mainly due to the two latter reasons as majority of O-SFGs are protonated. Highest enhancement of CEC in BC300 was achieved through NM due to the introduction of carboxylic groups. Though NM of BC500 showed a significant enhancement in CEC, the highest enhancement was observed via the HM. Since BC500 did not contain as much O-SFGs as BC300, pore opening and widening might have played a predominant role when determining CEC.

\section{Statistical analysis}

Due to small sample size $(n=3)$, a nonparametric ANOVA was conducted to find out whether there was a significant difference among the median value of a variable with respect to BC type. A Kruskal-Wallis test has been conducted. Significant differences were observed for the ash content of the raw, HMBC and SMBC with $p$-values of $0.027,0.026$, and 0.029 respectively. The difference in the ash content for NMBC was not statistically significant ( $p$-value $=$ 0.939). A significant linear relationship between the ash content and $\mathrm{BC}$ types for the modified BC was observed only for the HMBC.

Far from the normal BC types tested, for EC, the three different acid modified BC types showed a significant difference amongst them ( $p$-values $<0.05)$ whereas for the normal BC, the difference was not significant ( $p$ value $=0.055$ ).

There was a significant difference observed for the basic, acidic, lactonic and carboxylic groups present amongst the three types of normal $\mathrm{BC}$ ( $p$ value $=0.027$ ). The median of phenolic groups however did not differ significantly ( $p$-value $=0.051)$. For the two BC types which were subjected to acid modifications, the acidic, phenolic, lactonic and carboxylic moieties present differed significantly amongst them ( $p$-value $<0.05)$.

The CEC was observed to be different for BC300 and BC500 at $\mathrm{pH}$ 3, 7 and 10. Considering the same BC type at two different $\mathrm{pH}$ values, the values obtained were again different. The same pattern in results were got for the modified BCs as well although at $\mathrm{pH}$ 10, SM300 and SM500 showed no significant difference in CEC ( $p$-value $=0.077)$. Overall, there exists a significant difference in ash content, EC, acidic functional groups and CEC between the raw and modified $\mathrm{BC}$ types.
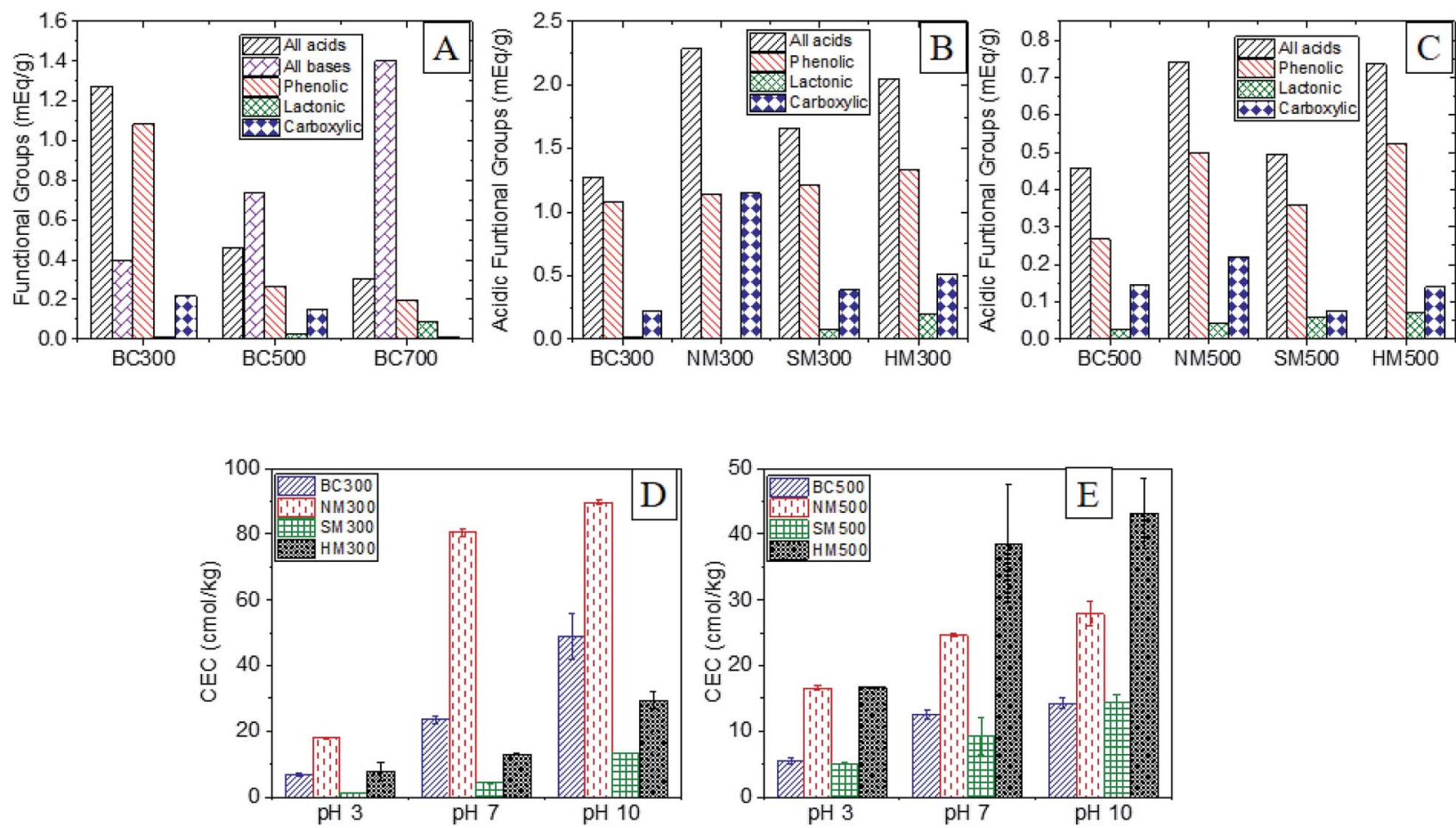

Fig. 5 Surface Functional Groups (SFGs) comparison for (A) raw BCs, (B) modified 300BCs, (C) modified 500BCs and CEC variation under different $\mathrm{pH}$ values (D) modified 300BCs and (E) modified 500BCs. 


\section{Conclusions}

Clear evidence exist to support the influence of acid modifications on physicochemical properties of the TWBC. Increasing pyrolysis temperature affects the surface acidity and the CEC as it can result in the decrement of acidic functionalities on the surface of the BC. The surface acidity for the raw BC samples were mainly as a contribution of phenolic groups. Incorporation of acid modifications yielded in elevated acidity for all the BC types tested. Far from the other modified samples which were in concordance with the raw BC showing insignificant variations in phenolic moieties, there was significant evidence that an enhancement in carboxylic groups contributed to the surface acidity upon NM. The SM, however, did not result in the formation of any carboxylic acid groups to a significant level but advanced the decarboxylation of the existing functionalities. The acid modified BC samples had low PZC values and showed a decrease in the ash content as a result of the demineralization and the introduction of acidic functionalities. The acidic PZC value of the $\mathrm{BC}$ becomes advantageous as it allows for the sorption of cationic species under mildly acidic conditions through electrostatic interactions. Oxygenated surface functional groups present on the $\mathrm{BC}$ surface have a notable contribution to CEC as well, especially at high pH. At basic pH, the functional moieties are deprotonated and surface complexation promoted. For the BC300, the highest CEC was observed when it was subjected to NM and for the BC500, the highest value was observed in HM.

When the pyrolysis temperature was increased, the aromaticity $(\mathrm{H} / \mathrm{C})$ and polarity index $(\mathrm{O}+\mathrm{N}) / \mathrm{C}$ decreased indicating enhanced aromatization and hydrophobicity of the $\mathrm{BC}$ that resulted at elevated temperatures. The XPS and FTIR results obtained for the raw and modified samples were in close agreement and it can be seen that the nitro and nitroxy atomic percentages have increased for the NMBC produced at higher temperatures as the nitration reaction becomes more favored. The $\mathrm{BC}$ produced at high temperature showed high thermal stability as confirmed from the TGA experiments.

The NM on TWBC can be used for agricultural experiments because of its high CEC to amend the nutrient leaching effect in soils. The SEM images of the SMBC show pore destruction which makes the retention of bulky adsorbents possible. However, to get an insight about these effects upon application of modified BC, it is necessary to conduct extensive field experimentation.

\section{Conflicts of interest}

There are no conflicts of interest to declare.

\section{Acknowledgements}

Authors would like to acknowledge the financial support by the Institute of Chemistry Ceylon (Research grant no 16-2). Authors would also like to acknowledge Sahan Jayasingha for the digital artwork.

\section{References}

1 M. Ahmad, A. U. Rajapaksha, J. E. Lim, M. Zhang, N. Bolan, D. Mohan, M. Vithanage, S. S. Lee and Y. S. Ok, Chemosphere, 2014, 99, 19-33.

2 A. G. Karunanayake, O. A. Todd, M. L. Crowley, L. B. Ricchetti, C. U. Pittman Jr, R. Anderson and T. E. Mlsna, Chem. Eng. J., 2017, 319, 75-88.

3 A. G. Karunanayake, O. A. Todd, M. Crowley, L. Ricchetti, C. U. Pittman Jr, R. Anderson, D. Mohan and T. Mlsna, Chem. Eng. J., 2018, 331, 480-491.

4 M. Uchimiya, L. H. Wartelle, K. T. Klasson, C. A. Fortier and I. M. Lima, J. Agric. Food Chem., 2011, 59, 2501-2510.

5 I. Herath, P. Kumarathilaka, A. Navaratne, N. Rajakaruna and M. Vithanage, J. Soils Sediments, 2015, 15, 126-138.

6 C. Peiris, S. R. Gunatilake, J. J. Wewalwela and M. Vithanage, in Biochar from Biomass and Waste, Elsevier, 2019, pp. 211224.

7 G. Xu, Y. Lv, J. Sun, H. Shao and L. Wei, Clean: Soil, Air, Water, 2012, 40, 1093-1098.

8 C. Peiris, S. R. Gunatilake, T. E. Mlsna, D. Mohan and M. Vithanage, Bioresour. Technol., 2017, 246, 150-159.

9 Y. Jayawardhana, S. R. Gunatilake, K. Mahatantila, M. P. Ginige and M. Vithanage, J. Environ. Manage., 2019, 238, 323-330.

10 A. Mukherjee, A. Zimmerman and W. Harris, Geoderma, 2011, 163, 247-255.

11 W. Zheng, B. K. Sharma and N. Rajagopalan, Using Biochar as a Soil Amendment for Sustainable Agriculture, Illinois Department of Agriculture Sustainable Agriculture Grant's Research Report Series, 2010.

12 D. Waters, J. Condon, L. Van Zwieten and S. Moroni, Biochar-ion interactions: an investigation of biochar charge and its effect on ion retention, 19th World Congress of Soil Science, Soil Solutions for a Changing World, Brisbane, Australia, 1-6 Aug 2010.

13 F. Aprile and R. Lorandi, J. Agric. Sci., 2012, 4, 278.

14 B. Liang, J. Lehmann, D. Solomon, J. Kinyangi, J. Grossman, B. O'neill, J. Skjemstad, J. Thies, F. Luizao and J. Petersen, Soil Sci. Soc. Am. J., 2006, 70, 1719-1730.

15 M. Uchimiya, I. M. Lima, K. Thomas Klasson, S. Chang, L. H. Wartelle and J. E. Rodgers, J. Agric. Food Chem., 2010, 58, 5538-5544.

16 J. Menendez, M. Illan-Gomez, C. L. y Leon and L. Radovic, Carbon, 1995, 33, 1655-1657.

17 O. D. Beltran-Pérez, A. Hormaza-Anaguano, B. Zuluaga-Diaz and S. A. Cardona-Gallo, Dyna, 2015, 82, 165-171.

18 C. Moreno-Castilla, M. Ferro-Garcia, J. Joly, I. BautistaToledo, F. Carrasco-Marin and J. Rivera-Utrilla, Langmuir, 1995, 11, 4386-4392.

19 J.-W. Shim, S.-J. Park and S.-K. Ryu, Carbon, 2001, 39, 16351642.

20 S. M. Yakout, A. E. H. M. Daifullah and S. A. El-Reefy, Environ. Eng. Manage. J., 2015, 14, 473-480.

21 D. K. Mahmoud, M. A. M. Salleh, W. A. W. A. Karim, A. Idris and Z. Z. Abidin, Chem. Eng. J., 2012, 181, 449-457. 
22 W. Shen, Z. Li and Y. Liu, Recent Pat. Chem. Eng., 2008, 1, 2740.

23 Y. Li, J. Shao, X. Wang, Y. Deng, H. Yang and H. Chen, Energy Fuels, 2014, 28, 5119-5127.

24 J. P. Chen and S. Wu, Langmuir, 2004, 20, 2233-2242.

25 G.-X. Yang and H. Jiang, Water Res., 2014, 48, 396-405.

26 B. B. Uzun, E. Apaydin-Varol, F. Ateş, N. Özbay and A. E. Pütün, Fuel, 2010, 89, 176-184.

27 A. Hicks, AU Journal of Technology, 2009, 12, 251-264.

28 https://intueriglobal.com/tea-industry-in-west-bengal/, accessed on March 31, 2019.

29 H. Dieng, R. B. M. Zawawi, N. I. S. B. M. Yusof, A. H. Ahmad, F. Abang, I. A. Ghani, T. Satho, H. Ahmad, W. F. Zuharah and A. H. Ab Majid, Ind. Crops Prod., 2016, 89, 157-166.

30 S. Cay, A. Uyanık and A. Özaşık, Sep. Purif. Technol., 2004, 38, 273-280.

31 S. Wan, Z. Ma, Y. Xue, M. Ma, S. Xu, L. Qian and Q. Zhang, Ind. Eng. Chem. Res., 2014, 53, 3629-3635.

32 A. U. Rajapaksha, M. Vithanage, M. Zhang, M. Ahmad, D. Mohan, S. X. Chang and Y. S. Ok, Bioresour. Technol., 2014, 166, 303-308.

33 M. Vithanage, S. Mayakaduwa, I. Herath, Y. S. Ok and D. Mohan, Chemosphere, 2016, 150, 781-789.

34 A. I. ASTM D2866-94(2004) Standard Test Method for Total Ash Content of Activated Carbon, West Conshohocken, PA, 2004, https://www.astm.org.

35 A. I. ASTM D2867-17 Standard Test Methods for Moisture in Activated Carbon, West Conshohocken, PA, 2017, https:// www.astm.org.

36 P. Liu, W.-J. Liu, H. Jiang, J.-J. Chen, W.-W. Li and H.-Q. Yu, Bioresour. Technol., 2012, 121, 235-240.

37 K. Yip, F. Tian, J.-i. Hayashi and H. Wu, Energy Fuels, 2009, 24, 173-181.

38 L. Tsechansky and E. Graber, Carbon, 2014, 66, 730-733.

39 J. W. Gaskin, C. Steiner, K. Harris, K. Das and B. Bibens, Trans. Am. Soc. Agric. Eng., 2008, 51, 2061-2069.

40 F. Ronsse, S. Van Hecke, D. Dickinson and W. Prins, GCB Bioenergy, 2013, 5, 104-115.

41 M. W. Schmidt, M. S. Torn, S. Abiven, T. Dittmar, G. Guggenberger, I. A. Janssens, M. Kleber, I. KögelKnabner, J. Lehmann and D. A. Manning, Nature, 2011, 478, 49.
42 J. Lehmann, J. Gaunt and M. Rondon, Mitigation and adaptation strategies for global change, 2006, vol. 11, pp. 403-427.

43 X. Cao and W. Harris, Bioresour. Technol., 2010, 101, 52225228.

44 D. B. Wiedemeier, S. Abiven, W. C. Hockaday, M. Keiluweit, M. Kleber, C. A. Masiello, A. V. McBeath, P. S. Nico, L. A. Pyle and M. P. Schneider, Org. Geochem., 2015, 78, 135-143.

45 A. V. McBeath, R. J. Smernik, M. P. Schneider, M. W. Schmidt and E. L. Plant, Org. Geochem., 2011, 42, 1194-1202.

$46 \mathrm{~J}$. Lehmann and S. Joseph, Biochar for Environmental Management: Science, Technology and Implementation, Taylor \& Francis, 2015.

47 C. E. Brewer, Biochar Characterization and Engineering, PhD thesis, Paper 12284, Iowa State University, 2012.

48 M. Ahmad, D. H. Moon, M. Vithanage, A. Koutsospyros, S. S. Lee, J. E. Yang, S. E. Lee, C. Jeon and Y. S. Ok, J. Chem. Technol. Biotechnol., 2014, 89, 150-157.

49 M. Ahmad, S. S. Lee, X. Dou, D. Mohan, J.-K. Sung, J. E. Yang and Y. S. Ok, Bioresour. Technol., 2012, 118, 536-544.

50 M. Ahmad, S. S. Lee, A. U. Rajapaksha, M. Vithanage, M. Zhang, J. S. Cho, S.-E. Lee and Y. S. Ok, Bioresour. Technol., 2013, 143, 615-622.

51 Z. Liu, W. Niu, H. Chu, T. Zhou and Z. Niu, Bioresources, 2018, 13, 3429-3446.

52 L.-L. Ling, W.-J. Liu, S. Zhang and H. Jiang, Environ. Sci. Technol., 2017, 51, 10081-10089.

53 M. Ahmad, S. S. Lee, S.-E. Oh, D. Mohan, D. H. Moon, Y. H. Lee and Y. S. Ok, Environ. Sci. Pollut. Res., 2013, 20, 8364-8373.

54 M. Vithanage, A. U. Rajapaksha, M. Zhang, S. Thiele-Bruhn, S. S. Lee and Y. S. Ok, Environ. Sci. Pollut. Res., 2015, 22, 2175-2186.

55 S.-X. Zhao, N. Ta and X.-D. Wang, Energies, 2017, 10, 1293.

56 A. Anstey, S. Vivekanandhan, A. Rodriguez-Uribe, M. Misra and A. K. Mohanty, Sci. Total Environ., 2016, 550, 241-247.

57 B. Chen, D. Zhou and L. Zhu, Environ. Sci. Technol., 2008, 42, 5137-5143.

58 D. Rehrah, M. Reddy, J. Novak, R. Bansode, K. A. Schimmel, J. Yu, D. Watts and M. Ahmedna, J. Anal. Appl. Pyrolysis, 2014, 108, 301-309.

59 B. Singh, M. Camps-Arbestain and J. Lehmann, Biochar: A Guide to Analytical Methods, CSIRO Publishing, 2017. 